\title{
Collision Induced Ion Ejection in an FTICR Trapped-Ion Cell
}

\author{
C Richard Arkin* and D. A. Laude \\ Department of Chemistry and Biochemistry, The University of Texas at Austin, Austin, Texas, USA
}

A collision algorithm was used with SimIon to evaluate collision-mediated ion ejection mechanisms in the ICR MS experiment. These mechanisms were characterized based on kinetic energy, ion mass, applied trapping potential, and collision gas mass. It was found that there are three collision-based energy regimes for ion loss from a trapped-ion cell. The first region is characterized by low initial cyclotron kinetic energy, a radial ejection mode, and a very high collision ratio ( $>100$ collisions per ejection). The second region is characterized by a medium to high initial cyclotron kinetic energy leading to axial ejection at low collision ratio ( 1 to 10 collisions per ejection). The third region is characterized by a high initial cyclotron kinetic energy, a radial ejection mode, and a collision ratio of unity. It was also determined that there is a radial cyclotron mode limit, approximately $40 \%$ of the cell radius, after which an ion is ejected after a single collision. This has important consequences on the damping of the FTICR signal, various cooling techniques, ion activation techniques, and the remeasurement experiment. (J Am Soc Mass Spectrom 2005, 16, 422-430) @ 2004 American Society for Mass Spectrometry

$\mathrm{T}$ Throughout the development of Fourier transform ion cyclotron resonance (FTICR) mass spectrometry, the mechanisms responsible for transient damping following excitation have been a significant source of study. There are four general mechanisms for transient damping. One is ion cloud broadening [1] resulting from collisions or inhomogeneties in the electric or magnetic fields [2]. Another mechanism is collisional stabilization of the cyclotron motion in which ions lose energy with minimal scattering and return to the center of the cell $[3,4]$. A third mechanism is the loss of signal that accompanies chemical reactions including charge transfer [5] or ion-molecule reaction. The fourth mechanism is ion ejection that can occur in either the axial [6] or radial $[7,8]$ direction. For many years the perception in the FTICR community was that the first two categories are the primary cause of signal damping of the FTICR transient. It is suggested here that for ions of moderate to high cyclotron kinetic energy (on the order of $10 \mathrm{eV}$ or greater in a $3 \mathrm{~T}$ magnetic field, for example) ion ejection is the primary mode of signal damping.

The first to discuss a mechanism for ion ejection following cyclotron excitation in a trapped-ion cell were Sharp et al. [7] who used a random walk model to describe a multiple collision ejection process. In this model, ions with relatively low initial cyclotron kinetic

Published online January 29, 2005

Address reprint requests to Dr. D. A. Laude, Department of Chemistry and Biochemistry (A5300), The University of Texas at Austin, Austin, TX 78712, U.S.A. E-mail: DALaude@mail.utexas.edu

* Current address: Hazardous Gas Detection Laboratory, ASRC Aerospace Corporation, ASRC-14, Kennedy Space Center, FL 32899, USA. energy undergo a large number of collisions, decreasing the cyclotron radius and changing the magnetron radius with each collision. Eventually, after a very large number of collisions, the ion is lost radially as a result of magnetron expansion. A second model suggested by Riegner and Laude [6] describes ions that achieve significant initial cyclotron kinetic energy and undergo relatively few collisions followed by axial ejection.

A collision algorithm [9] was used to investigate ion ejection mechanisms in FTICR trapped-ion cells. Two main parameters were studied-the mode of ejection, either axial or radial ejection, and the number of collisions that occurred prior to ejection. The percent axial ejection is used to describe the mode of ejection and is defined as the number of ions ejected axially divided by the total number of ions ejected. The collision ratio is used to quantify the number of collisions that occur per ejection and is defined as the total number of collisions that occur for each simulation experiment divided by the number of ions ejected. (All ions in the simulation are eventually ejected.) Using these two parameters it was found that there are three collision based energy regimes for ion ejection from a trapped-ion cell. The first region is characterized by low initial cyclotron kinetic energy, a radial ejection mode, and a very high collision ratio ( $>100$ collisions per ejection). This first region is consistent with that of the random walk model. The second region is characterized by a medium to high initial cyclotron kinetic energy leading to axial ejection at low collision ratio ( 1 to 10 collisions per ejection). This region is similar in behavior to that described by Reigner and Laude. The third region is characterized by a high initial cyclotron kinetic energy, 
a radial ejection mode and a collision ratio of unity. To our knowledge, this is the first description of this ion ejection mechanism.

\section{Collision Algorithm and Theory}

The algorithm described here is similar to the work of Lock and Dyer [10, 11] and specifically applies a collision model under FTICR conditions. The algorithm uses very basic concepts from the kinetic theory of gases. The particles are assumed to be hard spheres due to the high energies $(>10 \mathrm{eV})$ typically encountered in the FTICR experiment. The algorithm is Monte Carlo in nature in that it relies on calculating the probability of an ion-neutral collision and the probability of the glancing nature of the collision. The primary difference between this algorithm and the Lock and Dyer algorithm is reduced complexity. There are a variety of capabilities for simulating the FTICR experiment afforded by the algorithm. These capabilities include glancing collisions, magnetron growth beyond cyclotron radius, radial ejection, axial deflection, axial ejection, and collision with a neutral of same mass as the ion.

Briefly, as a simulation commences, SimIon will check to see if a user program exists after each time step and if so, it will execute the user program. (Although SimIon was the software package chosen for the simulations, the collision algorithm described here is generalized for use with other software packages.) The collision algorithm (the user program) will determine if a collision has occurred. If a collision has occurred, a subroutine will be entered that will determine the glancing conditions of the collision. By using two coordinate transformations, a one-dimensional attenuation of velocity is used to calculate the effect of a hard sphere collision under the glancing conditions. Two inverse coordinate transformations are used to return to the original frame of reference resulting in attenuated (postcollision) velocities. Thus a new velocity vector is substituted for pre-collision velocity vector. The collision algorithm assumes that the neutral is stationary with respect to the motion of the ion. How the collision affects the neutral is not calculated.

In more detail, the algorithm first determines the probability of a collision [12]. Eq 1 is used to calculate the probability, where $\lambda$ is the mean free path, and L is the

$$
\text { Probability }=1-e^{-\frac{\mathrm{L}}{\lambda}}
$$

distance the ion will travel in the next simulation time step. The mean free path is given in Eq 2 along with the ideal gas approximation, which shows the relationship between pressure, P, molecular diameter [13], $d$, temperature, $T$, the

$$
\lambda=\frac{1}{\sqrt{2} \pi d^{2} \bar{n}} \approx \frac{k T}{\sqrt{2} \pi d^{2} P}
$$

Boltzman constant, $\mathrm{k}=1.038 \times 10^{-5}$ torr $\cdot \AA^{2} \cdot \mathrm{m} \cdot \mathrm{K}^{-1}$, and mean free path. The algorithm then calls for a random number between 0 and 1 which is compared to the probability of collision. If the random number is greater than the probability of collision, nothing is done and the simulation is continued. If the random number is less than or equal to the probability for collision, then the program declares that a collision has occurred and enters the collision subroutine.

Now that a collision has occurred, the effect on the ion is calculated. There are two general methods to calculate the collisional effect. One is to perform a traditional conservation of momentum calculation with both the ion and the neutral in three dimensions. Or, a second is to perform coordinate transformations such that the vector describing the collision between the ion and the neutral corresponds to one of the component velocity vectors. There are two primary benefits if the second method is chosen. First, only a one-dimensional velocity attenuation needs to be calculated. Second, in performing the 1-dimensional velocity calculation, all calculations associated with the motion of the neutral may be ignored. (Since the motion of the neutral is not relevant to investigations of this kind, there is no need to calculate it.) The algorithm described here uses this

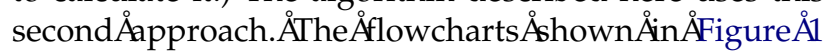
provide a graphical summary of the algorithm.

The first step is to perform a coordinate transform from the Cartesian coordinates to the velocity frame of reference (denoted by the prime superscript), which is

a)

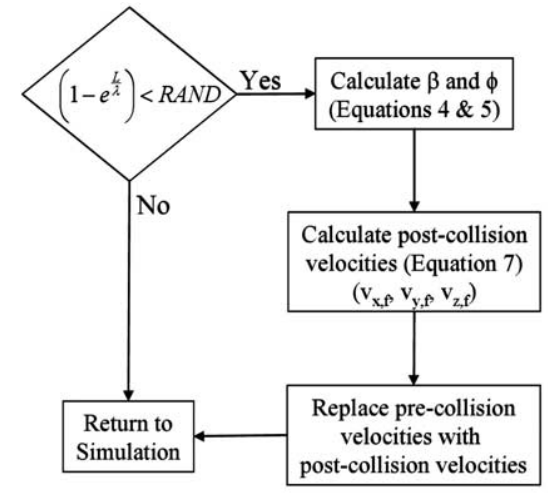

b)

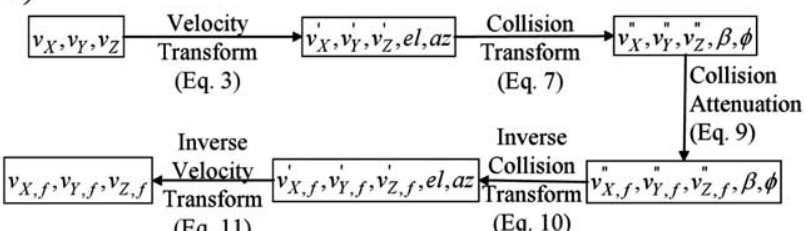

Figure 1. Flowchart of the collision algorithm. (a) Flowchart of the collision algorithm. (b) Flowchart describing how the postcollision velocities are calculated. 
given in eqs 3a-e. As can be seen from eqs $3 a-e$, the velocity frame of reference is essentially the spherical coordinate system; however, a different name and notation are used since the values for $\nu_{\mathrm{y}}^{\prime}$ and $\nu_{\mathrm{z}}^{\prime}$ will be non-zero after collision has occurred. The different name and notation are also used because SimIon data is in this format and it has an untraditional Cartesian to spherical transform (see eq 3e). (Note that SimIon provides the $v_{x}^{\prime}$ el, and az data, so this step is not required when using this simulation package. However, this step is described so that the algorithm may be generalized to any simulation package.)

$$
\begin{aligned}
& \nu_{\mathrm{x}}^{\prime}=\sqrt{\nu_{\mathrm{x}}^{2}+\nu_{\mathrm{y}}^{2}+\nu_{\mathrm{z}}^{2}} \\
& \nu_{\mathrm{y}}^{\prime}=0 \\
& \nu_{\mathrm{z}}^{\prime}=0 \\
& e l=\operatorname{Sin}^{-1}\left(\frac{\nu_{\mathrm{y}}}{\nu_{\mathrm{x}}^{\prime}}\right) \\
& \mathrm{az}=\operatorname{Tan}^{-1}\left(\frac{-\mathrm{v}_{\mathrm{z}}}{\mathrm{v}_{\mathrm{x}}}\right)
\end{aligned}
$$

Next, conversion to the collision frame of reference

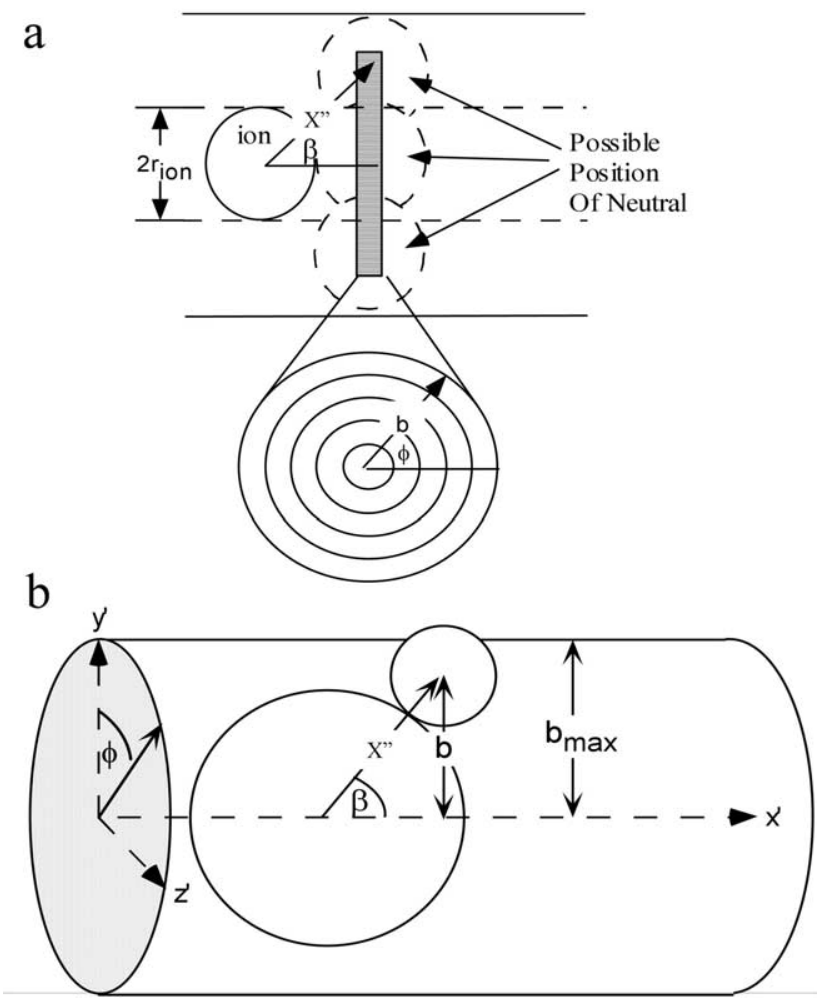

Figure 2. (a) Collision cross section and calculation of $\beta$-Pictorial representation describing the relationship between the first collision angle, $\beta$, the second collision angle, $\phi$, and the collision vector, $\nu_{\mathrm{x}}^{\prime \prime}$. (b) Definition of the parameters involved in a collision-Diagram of the ion and neutral colliding to form the collision vector, $\nu_{\mathrm{x}}^{\prime \prime}$ impact parameter, $\mathrm{b} \equiv \nu_{\mathrm{z}}^{\prime \prime}$, first collision angle, $\beta$, and second collision angle, $\phi$. must be performed. This step is performed so that the collision need only affect one vector, the $\nu_{x}^{\prime \prime}$ vector. In order to determine the relationship between the velocity frame of reference and the collision frame of reference, the glancing nature of the collision must be

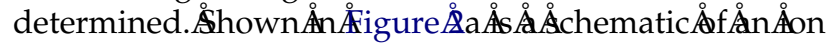
approaching several possible neutral positions. The probability of the angle of the neutral with respect to

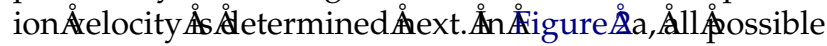
neutral angles are located on an imaginary disk. To determine the probability, the collision disk is subdivided into infinitesimally small rings of thickness $\mathrm{db}$. The probability, $\mathrm{P}(\mathrm{b})$, that the neutral center has an impact parameter of $b$ is the ratio of the area, $\pi b \cdot d b$, of the infinitesimal ring at $b$, to the area, $\pi b_{\max }^{2}$ of the collision cross-section (eq 4). Thus the probability of a direct hit in this model is zero and the probability is greatest for the most glancing (larger collision angle, $\beta$ ) collision. Also,

$$
\mathrm{P}(\mathrm{b}) \mathrm{db} \propto \frac{\pi \mathrm{bdb}}{\pi \mathrm{b}_{\max }^{2}} \propto \AA \AA
$$

the probability of the collision occurring at a collision disk radius $b$ is a linear function of $b$. To perform $a$ Monte Carlo simulation of this event, a linear random function generator is required, which can be produced by taking the square root of a constant probability

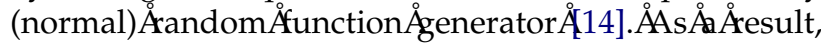
the first angle of the collision, $\beta$, is

$$
\begin{aligned}
\beta & =\operatorname{Sin}^{-1} \frac{b}{r_{\text {ion }}+r_{N}} \\
& =\operatorname{Sin}^{-1} \frac{\left(r_{\text {ion }}+r_{N}\right) \sqrt{\text { RAND }}}{r_{\text {ion }}+r_{N}} \\
& =\operatorname{Sin}^{-1} \sqrt{\text { RAND }}
\end{aligned}
$$

given by eq 5 where $r_{\text {ion }}$ and $r_{n}$ are the radii of the ion and neutral, respectively.

This method for calculating the $\beta$ angle of collision differs Årom $\AA$ hat

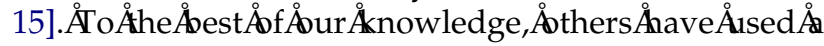
technique involving the production of two random numbers and choosing the larger of the two as the operand of the inverse sine for eq 5 . Both techniques produce a linear probability function, although the square root approach was chosen here since it is more efficient when writing software code.

The secondary angle for the neutral center, $\phi$, is determined. Since the probability of each angle is equal, a random number can be generated between zero and one, which is then multiplied by $2 \pi$ to determine the angle of collision. Thus the second angle of the collision is 


$$
\phi=2 \pi(\mathrm{RAND}) .
$$

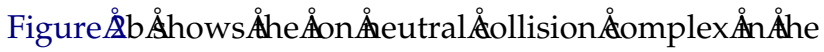
velocity coordinate system. The ion only has velocity along the $x^{\prime}$ axis. Upon conversion to the collision coordinates system, the $\mathrm{x}^{\prime \prime}$ axis will be directed from the center of the ion to the center of the neutral. This is accomplished by rotations about $\beta$ and $\phi$, and therefore conversion from the velocity frame of reference to the collision frame of reference (denoted by the double prime superscript) is given by eqs $7 \mathrm{a}-\mathrm{c}$.

$$
\begin{aligned}
\nu_{\mathrm{x}}^{\prime \prime} & =\nu_{\mathrm{x}}^{\prime} \cos \beta \cos \phi+\nu_{\mathrm{y}}^{\prime} \sin \beta \cos \phi+\nu_{\mathrm{z}}^{\prime} \sin \phi \\
& =\nu_{\mathrm{x}}^{\prime} \cos \beta \cos \phi \\
\nu_{\mathrm{y}}^{\prime \prime} & =-\nu_{\mathrm{x}}^{\prime} \sin \beta+\nu_{\mathrm{y}}^{\prime} \cos \beta=-\nu_{\mathrm{x}}^{\prime} \sin \beta \\
\nu_{\mathrm{z}}^{\prime \prime} & =-\nu_{\mathrm{x}}^{\prime} \cos \beta \sin \phi-\nu_{\mathrm{y}}^{\prime} \sin \beta \sin \phi+\nu_{\mathrm{z}}^{\prime} \cos \phi \\
& =-\nu_{\mathrm{x}}^{\prime} \cos \beta \sin \phi
\end{aligned}
$$

Now that the collision conditions have been determined, the result of the collision needs to be calculated. The most direct method is to calculate the velocity component in the direction of the collision (as was just performed) and then attenuate this value using the direct hit method using eq 8, where the initial velocity, $\nu$, is multiplied by the collision factor to give the final velocity, $\nu_{\mathrm{f}}$. In order to determine the post-collision

$$
\nu_{f}=\left(\frac{m_{i o n}-m_{n}}{m_{\text {ion }}+m_{n}}\right) \nu
$$

(a)

(b)
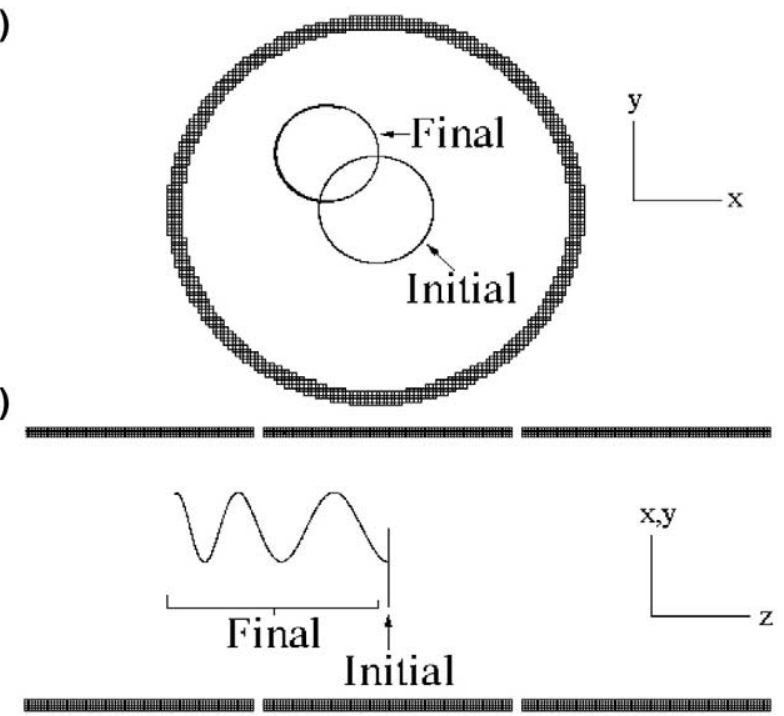

Figure 3. A collision as it occurs in SimIon. A $2 \mathrm{kDa}$ ion with only cyclotron motion initially (30\% cell radius). Following collision with helium, the (a) cyclotron radius is reduced, the magnetron radius is increased as is the (b) axial amplitude. This figure illustrates the glancing nature of this algorithm. velocity in the collision system, eqs $9 \mathrm{a}-\mathrm{c}$ are used. Now that the velocity has been attenuated, the velocity vector in the Cartesian frame of reference needs to be determined.

$$
\begin{aligned}
& \nu_{\mathrm{x}, \mathrm{f}}^{\prime \prime}=\left(\frac{m_{\text {ion }}-m_{n}}{m_{\text {ion }}+m_{n}}\right) \nu_{\mathrm{x}}^{\prime \prime}=\left(\frac{m_{\text {ion }}-m_{n}}{m_{\text {ion }}+m_{n}}\right) \cdot \nu_{\mathrm{x}}^{\prime} \cos \beta \cos \phi \\
& \nu_{\mathrm{y}, \mathrm{f}}^{\prime \prime}=\nu_{\mathrm{y}}^{\prime \prime}=-\nu_{\mathrm{x}}^{\prime} \sin \beta \\
& \nu_{\mathrm{z}, \mathrm{f}}^{\prime \prime}=\nu_{\mathrm{z}}^{\prime \prime}=-\nu_{\mathrm{x}}^{\prime} \cos \beta \sin \phi
\end{aligned}
$$

Returning from the collision coordinate system to the velocity frame of reference is accomplished using eqs $10 \mathrm{a}-\mathrm{c}$. The transform from the velocity coordinate system to Cartesian coordinates

$$
\begin{aligned}
& \mathrm{v}_{\mathrm{x}}^{\prime}=\mathrm{v}_{\mathrm{x}}^{\prime \prime} \cos \beta \cos \phi-\mathrm{v}_{\mathrm{y}}^{\prime \prime} \sin \beta-\mathrm{v}_{\mathrm{z}}^{\prime \prime} \cos \beta \sin \phi \\
& \mathrm{v}_{\mathrm{y}}^{\prime}=\mathrm{v}_{\mathrm{x}}^{\prime \prime} \sin \beta \cos \phi+\mathrm{v}_{\mathrm{y}}^{\prime \prime} \cos \beta-\mathrm{v}_{\mathrm{z}}^{\prime \prime} \sin \beta \sin \phi \\
& \nu_{\mathrm{z}}^{\prime}=\nu_{\mathrm{x}}^{\prime \prime} \sin \phi+\nu_{\mathrm{z}}^{\prime \prime} \cos \phi
\end{aligned}
$$

is accomplished using eqs $11 \mathrm{a}-\mathrm{c}$.

$$
\begin{aligned}
& \nu_{\mathrm{x}}=\nu_{\mathrm{x}}^{\prime} \cos (e l) \cos (a z)-\nu_{\mathrm{y}}^{\prime} \sin (e l)-\nu_{\mathrm{z}}^{\prime} \cos (e l) \sin (a z) \\
& \nu_{\mathrm{y}}=\nu_{\mathrm{x}}^{\prime} \sin (e l) \cos (a z)+\nu_{\mathrm{y}}^{\prime} \cos (e l)-\nu_{\mathrm{z}}^{\prime} \sin (e l) \sin (a z) \\
& \mathrm{v}_{\mathrm{z}}=\mathrm{v}_{\mathrm{x}}^{\prime} \sin (\mathrm{az})+\mathrm{v}_{\mathrm{z}}^{\prime} \cos (\mathrm{az})
\end{aligned}
$$

By combining eqs 3, 9, 10, and 11 and simplifying, the post-collision velocity component vectors in Cartesian coordinates

$$
\begin{aligned}
\mathrm{v}_{\mathrm{x}, \mathrm{f}}= & \mathrm{v}_{\mathrm{x}}^{\prime}\{[\cos \beta \cos \phi \cos (e l) \cos (a z) \\
& -\sin \beta \cos \phi \sin (e l) \\
& -\sin \phi \cos (e l) \sin (a z)] \mathrm{F} \cos \beta \cos \phi \\
& +\cos (e l) \cos (a z)\}
\end{aligned}
$$

$$
\begin{aligned}
\nu_{\mathrm{y}, \mathrm{f}}= & \mathrm{v}_{\mathrm{x}}^{\prime}\{[\sin \beta \cos \phi \cos (e l)-\sin \phi \sin (e l) \sin (a z) \\
& -\cos \beta \cos \phi \sin (e l) \cos (a z)] \mathrm{F} \cos \beta \cos \phi \\
& +\sin (e l) \cos (a z)\}
\end{aligned}
$$

$$
\begin{aligned}
\nu_{z, \mathrm{f}}= & \mathrm{v}_{\mathrm{x}}^{\prime}\{[\cos \beta \cos \phi \sin (a z) \\
& +\sin \phi \cos (a z)] \mathrm{F} \cos \beta \cos \phi+\sin (a z)\}
\end{aligned}
$$

where $\mathrm{F} \equiv\left(\frac{\mathrm{m}_{\text {ion }}-\mathrm{m}_{\mathrm{n}}}{\mathrm{m}_{\mathrm{ion}}+\mathrm{m}_{\mathrm{n}}}\right)-\AA . \AA$ A Figure $\AA \AA$ Shows $\AA$ SimIon 
screenshot of the collision algorithm in use, where an ion has only cyclotron motion initially, but after a collision, posses all three modes of motion-cyclotron, magnetron, and axial.

\section{Experimental}

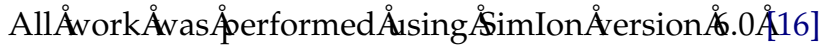
on a $500 \mathrm{MHz}$ AMD PC computer. A LabVIEW (National Instruments, Austin, TX) program was written to extract and process the data generated by SimIon.

All simulation models were derived from geometry files for accurate reproduction of the open cylindrical trapped-ion cell and vacuum chamber under investigation. The trapped-ion cell had an aspect ratio of 1.8, i.d. of $102 \mathrm{~mm}$, excite/detect electrode length of $90 \mathrm{~mm}$, an electrode gap of $4 \mathrm{~mm}$, and trap electrode length of 90 $\mathrm{mm}$. The vacuum chamber had an i.d. of $160 \mathrm{~mm}$ and length of $350 \mathrm{~mm}$.

Unless otherwise stated, all simulation experiments used ions of unit charge and a neutral collision gas mass of $4 \mathrm{Da}$. The diameter of the ions and neutrals was assumed to be $7.5 \AA$ for mean-free-path calculations. Typically 100 ions were used in each experiment in an attempt to approach a statistical number of ions while still being time efficient. The initial kinetic energy of the ion was determined such that prior to a collision the ion would only have cyclotron motion. Unless otherwise noted, experiments were conducted at high, simulated pressures $\left(5 \times 10^{-6}\right.$ torr $)$ to reduce calculation times. Ten volts were applied to the trapping electrodes and the excite/detect electrodes were grounded. A homogenous magnetic field of 3 tesla was superimposed in the axial direction of the simulation. Computation times varied from a few minutes to several hours. The primary factor that affected computation times was the energy (or cyclotron radius) of the ion under investigation.

\section{Results and Discussion}

Given sufficient time, any ion will eventually be lost from a trapped-ion cell. The mechanism investigated here is collisional ion loss, which is the primary mechanism in most FTICR experiments.

\section{Description of the Three Collisional Ejection Regions}

Using SimIon and the collision algorithm, it was indicated that there are three general mechanisms that

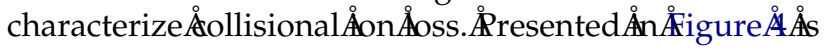
a graphical representation of ion ejection for a $1 \mathrm{kDa}$ ion in a $3 \mathrm{~T}$ magnetic field. There are two plots: an ion loss curve and a collision ratio curve. The ion loss curve gives the route by which an ion is lost from the cell. For

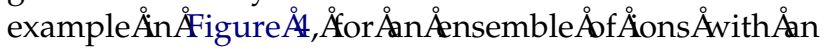
initial cyclotron radius of $20 \%$, all of these ions will be

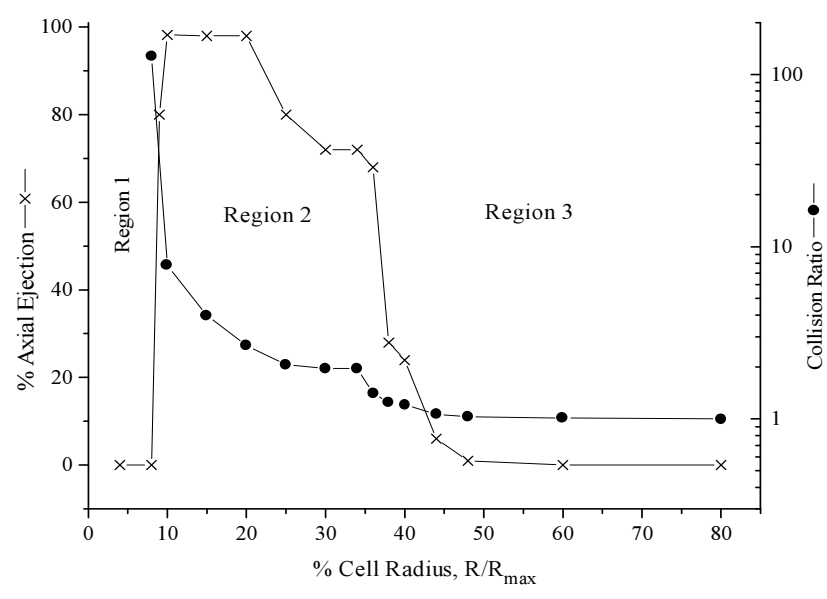

Figure 4. Illustration of the three ejection regions. The first, the random walk region, is characterized by radial ejection and a high collision ratio. The second region is characterized by axial ejection and a near unit collision ratio. The third region is characterized by radial ejection and a unit collision ratio.

ejected axially and none will be ejected radially. Yet, at an initial cyclotron radius of $40 \%$, an increase in radial ejection occurs; $20 \%$ are ejected axially and $80 \%$ are ejected radially. The collision ratio curve describes the average number of collisions an ion has with a neutral prior to ejection. Furthermore, at an initial radius of $20 \%$, each ion will undergo approximately 2 collisions prior to ejection. For an initial radius of $40 \%$, each ion will undergo an average of 1.03 collisions prior to ejection.

Using these two types of information in tandem, ion loss can be placed in three categories. The first category is the well-known random walk mechanism. It is characterized here by a radial ejection mechanism that occurs at high collision ratio $(>100)$. This category is labeled $\AA$ s̊Region $\AA$ Af Figure $\AA$.

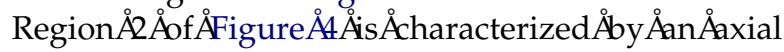
ejection mechanism and a low collision ratio (1 to 10). RiegnerÅnd ÅLaude $\AA$ [6] Ånitially Ådentified Ånd Åxperimentally observed this collision process. For this mechanism, the ion has sufficient radial kinetic energy that after a few collisions, sufficient kinetic energy has been transferred to the axial mode of motion such that the ion may escape the relatively low z-axis trapping potential depression. The boundary between Regions 1 and 2 is dependent on several factors including trap potential and the mass of the collision gas and ion.

A radial ejection mechanism that occurs at a collision

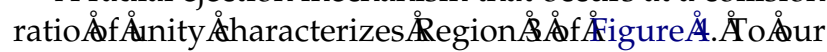
knowledge, there has never been any discussion on this ejection process. It is actually an extension of Region 2 in that the ion has sufficient axial kinetic energy to overcome the trapping potential. The reason that the ejection mode is radial is that substantial kinetic energy has also been transferred to the magnetron mode. As a result, there is a large magnetron radius that permits the ion to collide with the cell wall. The radial ejection mode is more likely than the axial ejection process 


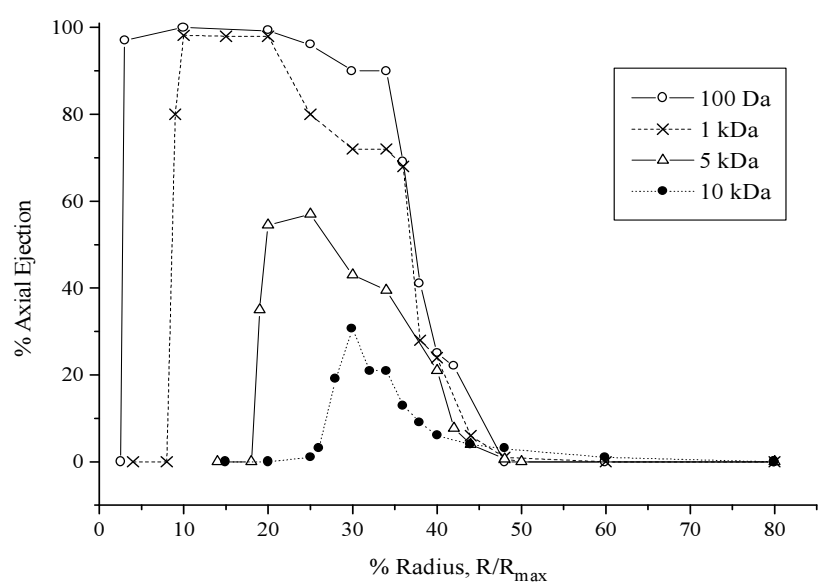

Figure 5. Mass dependence on the collision mechanisms. Ion loss curves for several different masses under otherwise similar conditions including a collision gas mass of $4 \mathrm{Da}$, an applied potential of $10 \mathrm{~V}$, and a magnetic field of $3 \mathrm{~T}$. Each transition between Regions 1 and 2 occurs at approximately $9 \mathrm{eV}$ and the boundary between Regions 2 and 3 is approximately $40 \%$ of the radius.

because the cyclotron motion velocity is higher than the axial ejection velocity, typically by 2 orders of magnitude. It must be emphasized that the ejection mechanism for ions in Region 3 occurs after a single collision and then is ejected in less than one-half of a cyclotron revolution. The boundary between Regions 2 and 3 is stable at approximately $40 \%$ the cell radius, and independent of ion mass, collision gas mass and trap potential.

The cyclotron radius for the onset of Region 3 onset has a theoretical minimum of $1 / 3 \mathrm{r}_{\max }$. This is because, if the collision causes the ion to maintain essentially the same cyclotron radius and the magnetron radius increases to the previous cyclotron radius, then ejection will occur. This $33 \%$ cell radius is a minimum boundary condition, yet the actual Region 3 boundary will be higher since not all ion-neutral collisions will be direct, elastic collisions.

\section{Mass Dependence of Collision Mechanisms}

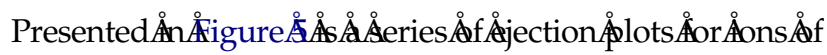
varying mass. The three ejection regions are present for each mass. The boundary between Regions 1 and 2 is mass dependent. For increasing mass, the cell radius that defines the boundary increases. The boundaries for 100, 1000, 5000, and 10,000 Da are approximately 3, 8, 17, and 27 , respectively. Although the initial cyclotron radius is different for each of these masses, the initial cyclotron kinetic energy is constant at approximately $9 \mathrm{eV}$.

A second mass-dependent feature of collision is the reduction in axial ejection with increasing mass in Region 2. This is observed directly from the collision factor in eq 8. With a constant neutral mass, $m_{n}$, an increasing ion mass, $m_{i o n}$, results in

$$
v_{f}=\left(\frac{m_{i o n}-m_{n}}{m_{\text {ion }}+m_{n}}\right) v
$$

a collision factor that approaches unity and a postcollision velocity, $v_{f}$, that approaches the pre-collision velocity, $\mathrm{v}$. When the collision factor approaches unity much less relative energy is removed per collision, and consequently high mass ions need more collisions to be ejected. For each collision, the magnetron radius is increased, and a mechanism similar to that of Region 1 results. After two or more collisions, the magnetron radius has increased to the extent such that the cyclotron motion intersects the cell.

It is important to point out that throughout the literature, it has been the consensus that only low mass

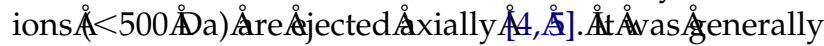
thought that if the collision factor was near unity $\left(\mathrm{M}_{\mathrm{ion}}\right.$ $\gg M_{n}$ ) then ions would relax to the center of the cell.

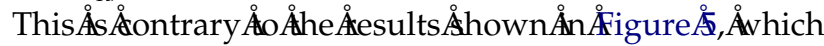
shows axial ejection up to mass $10 \mathrm{kDa}$.

a)

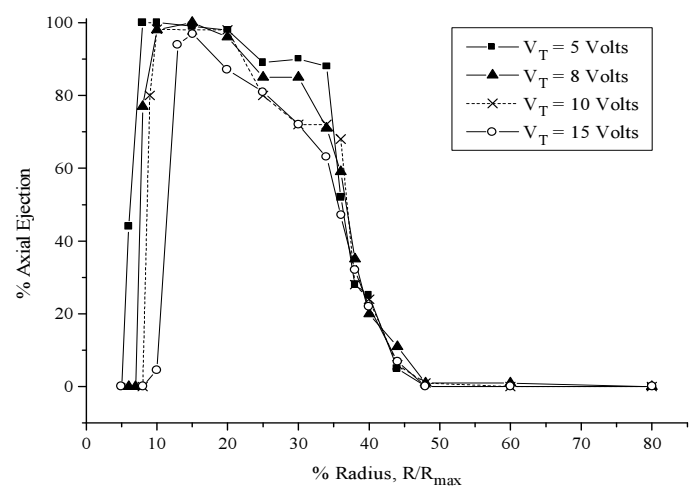

b)

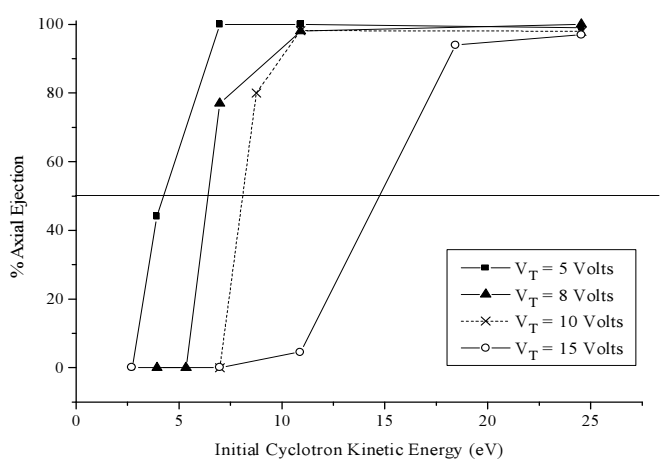

Figure 6. Applied trapping potential dependence on ejection mechanisms. The applied trapping potentials are varied from 5 to $15 \mathrm{~V}$ while the ion mass, collision gas mass, and magnetic field are constant at $1 \mathrm{kDa}, 4 \mathrm{Da}$, and 3T, respectively. (a) The boundary between Regions 1 and 2 is a function of applied potential, but the boundary between Regions 2 and 3 is approximately $40 \%$ of the cell radius and is independent of applied potential. (b) A closer examination of the boundary between Regions 1 and 2 as a function of axial kinetic energy reveals that on average the minimum ejection energy is $69 \%$ of the applied trapping potential. 


\section{Trap Potential Dependence on Collision Mechanisms}

Presented ̊̊n Firigure $\mathrm{kDa}$ ion with varying potentials applied to the trapping electrodes. There are two primary features of interest. First, the boundary between Region 2 and 3 is independent of the potential applied to the trapping electrodes. Second, the boundary between Region 1 and Region 2 is dependent on the trapping potential. This dependency

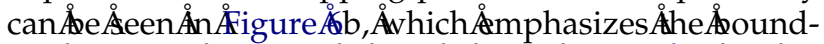
ary $x$-axis transformed to initial cyclotron kinetic energy instead of cyclotron radius. The kinetic energy, KE, was

$$
\mathrm{KE}=\frac{\mathrm{q}^{2} \mathrm{~B}^{2} \mathrm{r}^{2}}{2 \mathrm{~m}}
$$

calculated Å[17]Åusing ÅtheÅrelationship Åbetween Åion charge, $\mathrm{q}$, ion mass, $\mathrm{m}$, magnetic field, $\mathrm{B}$, and cyclotron radius, $r$, as described by eq 13 .

Using SimIon, it was determined that the centerline well depth of this cell geometry was $0.693 \mathrm{~V} / \mathrm{V}_{\text {appli }}$; therefore when $5 \mathrm{~V}$ is applied to the trapping electrodes an ion would need a minimum of $3.5 \mathrm{eV}$ of axial kinetic energy to leave the cell. The minimum ejection energy for 8,10 and $15 \mathrm{~V}$ applied is $5.5,6.9$, and $10.4 \mathrm{eV}$, respectively. This corresponds to $68.8,69.0$, and $69.3 \%$ of the potential energy well depth, respectively. On average, this ejection energy is $69 \%$ of the applied trapping potential, which is consistent with the theoretical minimum of $69.3 \%$.

\section{Collision Gas Mass Dependence on Collision Mechanisms}

The attenuated velocity for a glancing collision is determined by eq 8 . It is the attenuated velocity that determines the kinetic energy loss from the ion, and thus the mode of ejection. Since the mass of the collision gas, $\mathrm{m}_{n}$ is a factor in eq 8 , it is expected that collision gas mass should affect the ejection loss curves. Presented in

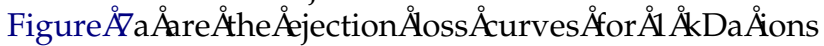
colliding with neutrals of 4, 40, and $131 \mathrm{Da}$. The boundary between Regions 1 and 2 are dependent on the mass of the collision gas and moves to higher ion radius with increasing collision gas mass. The boundary between Regions 2 and 3 remains constant at approxi-

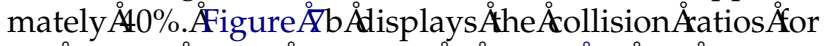

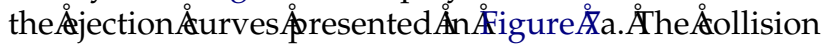
ratio curve supports the ejection curve data that the boundary between Regions 1 and 2 is mass dependent.

\section{Upper Mass Limit on Region 2}

Since the boundary between Regions 1 and 2 is mass dependent, there will be a mass where the boundary between Regions 1 and 2 equals the boundary between Regions 2 and $3(\sim 40 \%)$. This mass is the theoretical a)

b)

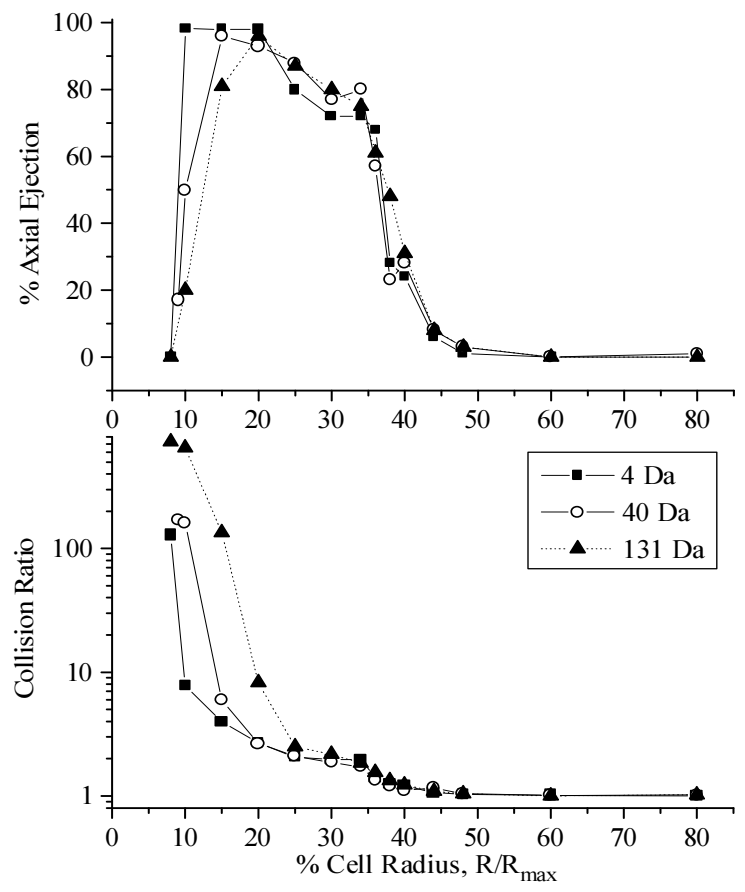

Figure 7. Collision gas mass dependence on ejection mechanisms. Using collision gas masses of 4, 40, and $131 \mathrm{Da}$ with an ion of $1 \mathrm{kDa}$, it is shown that the boundary between Regions 1 and 2 is dependent on the mass of the collision gas, and the boundary between Regions 2 and 3 is independent. (a) Neutrals of greater mass shift the first boundary to higher cell radius, while the second boundary remains constant at approximately $40 \%$ of the cell radius, based on ion loss curves. (b) Collision ratio curves support the ion loss data.

upper mass limit for Region 2. This mass can be calculated from the kinetic energy, KE, of the boundary between Regions 1 and 2, the cell radius, r, of the boundary between Regions 2 and 3, and using eq 14, where $B$ is the magnetic field strength and $q$ is the charge of the ion. For example in the cell used here, $r$ is $19.38 \mathrm{~mm}$, and with $1 \mathrm{~V}$ applied to the trapping electrodes, $\mathrm{KE}$ is

$$
\mathrm{m}_{\text {upper }}=\left(4.824 \times 10^{7} \frac{\mathrm{eV} \cdot \mathrm{Da}}{\mathrm{e}^{2} \cdot \mathrm{T}^{2} \cdot \mathrm{m}^{2}}\right) \frac{\mathrm{q}^{2} \mathrm{~B}^{2} \mathrm{r}^{2}}{\mathrm{KE}}
$$

$0.86 \mathrm{eV}$. With a single charge and a magnetic field of 3 tesla, the upper mass limit is $190 \mathrm{kDa}$. Under these conditions, for masses greater than $190 \mathrm{kDa}$, there is no mechanism available for axial ejection. When $10 \mathrm{~V}$ is applied to the trapping electrodes, the kinetic energy for the boundary between Regions 1 and 2 is $8.6 \mathrm{eV}$ resulting in an upper mass limit of $19 \mathrm{kDa}$.

\section{Revised Collisional Damping Model for High Energy Ions}

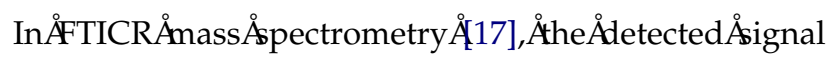
results in the production of a transient. Such a transient 
is $\operatorname{modeled}$ hy The damping term is the result of a variety of processes including ÅcollisionalÅdamping $\AA[3, \AA 34]$, Åspace $̊$ charge

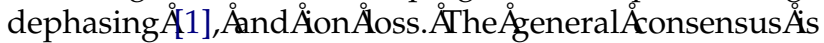
that when experimental conditions are optimized, the signal damping is only a result of collisional damping and dephasing.

From the data presented here, specifically the unit collision ratio ejection that occurs throughout Region 3 , there is evidence that the line of thought that ions collisionally relax back to the center of the cell or dephaseÅs hot Åalid. F̂igure up to a mass of $10 \mathrm{kDa}$ colliding with a neutral of $4 \mathrm{Da}$, which clearly meets the criterion of $\mathrm{M}_{\mathrm{ion}} \gg \mathrm{M}_{\text {neutral }}$ of

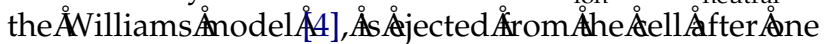
collision with a neutral. Thus, the primary cause for the damping of the transient at high kinetic energy, Region 3 , is ion ejection.

The mechanism of radial ion loss in Region 3 was discussed above, but the time scale of ion loss can be calculated from collision theory as well. The mean free path, $\lambda$, for a particle is given by eq 2 . Assuming a temperature of $300 \mathrm{~K}$ and a molecular diameter of $10 \AA$, pressures of $1 \times 10^{-8}, 5 \times 10^{-8}, 1 \times 10^{-7}$, and $5 \times 10^{-7}$ torr result in mean free paths of 702, 140, 70, and $14 \mathrm{~m}$, respectively. The probability the particle will collide with another particle is given by eq 15 where $\mathrm{L}$ is the distance the particle travels, $\lambda$ is the mean free path, $\mathrm{v}$ is the velocity of the particle and $t$ is time. The probability

$$
\text { Probability }=1-\exp \left(-\frac{\mathrm{L}}{\lambda}\right)=1-\exp \left(-\frac{\mathrm{v} \cdot \mathrm{t}}{\lambda}\right)
$$

can also be interpreted to mean the percent of ions to experience a collision. For example, $63 \%$ of all ions should have a collision after traveling a distance equal to the mean free path. The velocity is determined using eq 16 where $\mathrm{KE}$ is the kinetic energy and $\mathrm{m}$ is mass.

$$
\mathrm{v}=\left(13.891 \frac{\mathrm{Da}^{\frac{1}{2}} \cdot \mathrm{m}}{\mathrm{eV}^{\frac{1}{2}} \cdot \mathrm{ms}}\right) \sqrt{\frac{\mathrm{KE}}{\mathrm{m}}}
$$

For an example, a $1 \mathrm{kDa}$ ion with an initial cyclotron radius of $48 \%$ cell radius, which corresponds to 24.48 $\mathrm{mm}$ and $252 \mathrm{eV}$ under the conditions discussed here, is chosen. Under these conditions, the ion has a velocity of $6.96 \mathrm{~m} / \mathrm{ms}$. With this velocity about $63 \%$ of the ions should have a collision within $100 \mathrm{~ms}$ at a pressure of $1 \times 10^{-8}$ torr. Because these ions are Region 3 ions, this means that about $63 \%$ of the ions have been ejected $100 \mathrm{~ms}$ after the excitation event. This time scale may be longer than many transients for a single scan, but this has serious ramifications for the remeasurement, activation and other ion manipulation experiments. Using the same conditions but with a pressure of $1 \times 10^{-7}$ torr, about $63 \%$ of the ions should be ejected within $10 \mathrm{~ms}$. This detection time scale is not desirable in FTICR.

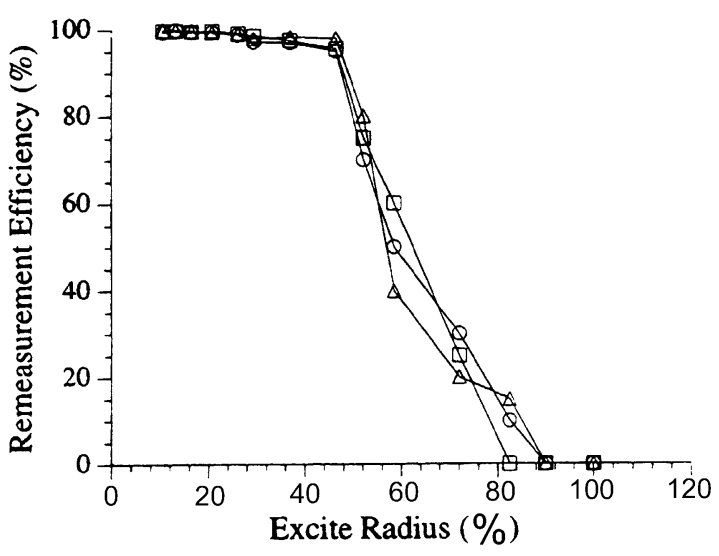

Figure 8. Remeasurement with QE. Even with active ion cooling there is still a maximum excitation radius near $46 \%$ of the cell radius. Shown here are the experimental remeasurement efficiencies for the +4 charge state of melittin (open circle), the +5 charge state of insulin (open square), and the +15 charge state of horse heart myoglobin (open triangle) as adapted from reference [18]. This provides experimental validation of the various ejection mechanisms discussed. [Reproduced with permission from Anal. Chem. 1995, 67, 1717-1721. Copyright 1995 Am. Chem. Soc.]

\section{Quadrupolar Excitation (QE) Remeasurement}

It ̊̊vas ̊̊demonstrated $\AA$ by $\AA$ Hendrickson $\AA$ nd $\AA$ Laude $\AA 2[18]$ that when quadrupolar excitation (QE) is used for remeasurement, near unit remeasurement efficiency can be achieved for a ion radius ranging up to approximately $45 \%$ of the cell radius. Beyond that radius, the remeasurement efficiency declines to near zero by $80 \%$

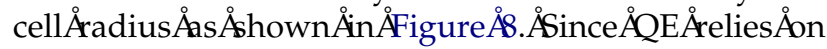
collisional dampening of the cyclotron motion following conversion of the magnetron motion to cyclotron motion, it is important to discuss QE remeasurement in terms of the three regions of ion ejection developed here. Since remeasurement occurs over a long time scale there is ample time for ion loss by all three mechanisms discussed.

Region 1 is the random walk region where there are hundreds of collisions and the ions slowly expand radially. When ions are in this region, $\mathrm{QE}$ is effective because the ions are axialized much faster than they are ejected since so many collisions are involved for cooling to occur. Region 2 typically involves two or more collisions prior to axial ejection under normal experimental conditions. [During a QE event, the pressure is elevated to relatively high pressure such as $1 \times 10^{-5}$ torr where the mean free path of an ion with a molecular diameter of $10 \AA$ is $70 \mathrm{~cm}$.] At a cyclotron radius consistent with Region 2, an ion would have so many collisions prior to axial ejection, that the ion would have cooled down to become a Region 1 ion. For these reasons QE works well for both Regions 1 and 2, or for a cell radius up to approximately $40 \%$ according to the hard sphere model used here. In contrast, ions in Region 3 will still be ejected immediately after the first collision even during the $\mathrm{QE}$ event. This is the reason that the 
remeasurement efficiency for ions at large excitation

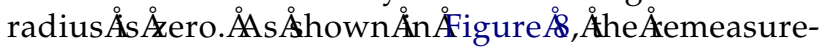
ment efficiency does not decline to zero until an excitation radius of about $80 \%$. A possible reason is that the ions in the experiment are not hard spheres and do not undergo elastic scattering.

\section{Conclusions}

Using ion simulations with a collision algorithm, three separate mechanisms of ion loss were described. The first mechanism, Region 1, involves ions at small initial cyclotron radius that are ejected radially after being involved with a large number of collisions. The Region 2 mechanism involves ions of moderate cyclotron radius. Ions undergoing collisions under these conditions are ejected axially after undergoing relatively few (one to ten) collisions. Region 3 involves ions of large cyclotron radius and correspondingly high kinetic energy. Such ions are ejected radially after only a single collision. The boundary between Region 1 and 2 is dependent on collision gas mass, ion mass and trapping potential. The boundary between Regions 2 and 3 is fixed at approximately $40 \%$. These mechanisms were used to explain the experimentally observed remeasurement efficiency.

\section{References}

1. Grosshans, P. B.; Marshall, A. G. Can Fourier transform mass spectral resolution be improved by detection at harmonic multiples of the fundamental ion cyclotron orbital frequency? Int. J. Mass Spectrom. Ion Processes 1991, 107, 49-81.

2. Anderson, S. A. Development and evaluation of a low homogeneity magnet for FTICR mass spectrometry; Ph.D. Dissertation, The University of Texas at Austin, December 1996.

3. Comisarow, M. B. Signals, noise, sensitivity, and resolution in ion cyclotron resonance spectroscopy in ion cyclotron spectrometry, II. Lecture notes in chemistry. Hartmann, H. J.;
Wanczek, K. P., Eds.; Springer-Verlag: New York, 1982; pp 484-513.

4. Williams, E. R.; Henry, K. D.; McLafferty, F. W. Multiple remeasurement of ions in Fourier transform mass spectrometry. J. Am. Chem. Soc. 1990, 112, 6157-6162.

5. Beauchamp, J. L. Theory of collision broadened ICR spectra. J. Chem. Phys. 1967, 46, 1231-1243.

6. Riegner, D. E.; Laude, D. A. Collision-mediated axial ejection of radially excited ions as a mass-dependent ion loss mechanism in Fourier transform ion cyclotron mass spectrometry. Int. J. Mass Spectrom. Ion Processes 1992, 120, 103-116.

7. Sharp, T. E.; Eyler, J. R.; Li, E. Electrostatic potential for an orthorhombic ion trap. Int. J. Mass Spectrom. Ion Phys. 1972, 9, 421-439.

8. Beu, S. C.; Laude, D. A. Radial ion transport due to resistivewall destabilization in Fourier transform mass spectrometry. Int. J. Mass Spectrom. Ion Processes 1991, 108, 255-268.

9. Arkin, C R. Theoretical Treatment of Ion Motion in an Open Trapped-ion Cell for use in Fourier Transform Ion Cyclotron Resonance Mass Spectrometry; Ph.D. Dissertation, The University of Texas at Austin, August 2000.

10. Lock, C. M.; Dyer, E. W. Simulation of ion trajectories through a high pressure radio frequency only quadrupole cell by SIMION 6.0. Rapid Commun. Mass Spectrom. 1999, 13, 422-431.

11. Lock, C. M.; Dyer, E. W. Characterization of high pressure quadrupole collision cells possessing direct current axial fields. Rapid Commun. Mass Spectrom. 1999, 13, 432-448.

12. Present, R. D. Kinetic theory of gases; McGraw-Hill: NewYork, 1958; p 29

13. CRC Handbook of Chemistry and Physics, 70th ed; Weast, R. C., Ed.; CRC Press: Boca Raton, FL, 1989 p D-90.

14. Gentle, J. E. Random number generation and Monte Carlo methods; Springer-Verlag: New York, 1998; pp 96-97

15. Douglas, D. J.; French, J. B. Collisional focusing effects in radio frequency quadrupoles. J. Am. Soc. Mass Spectrom. 1992, 3, 398-408.

16. Dahl, A. D. SimIon3D version 6.0. Proceedings of the $43 \mathrm{rd}$ ASMS Conference on Mass Spectrometry and Allied Topics; May 1995; p 717.

17. Marshall, A. G.; Hendrickson, C. L.; Jackson, G. S. Fourier transform ion cyclotron resonance mass spectrometry: A Primer. Mass Spectrom. Rev. 1998, 17, 1-35.

18. Hendrickson, C. L.; Laude, D. A. Quadrupolar axialization for improved control of electrosprayed proteins in FTICR mass spectrometry. Anal. Chem. 1995, 67, 1717-1721. 